\title{
High Resolution Secondary-Electron and ADF-STEM Imaging with a Hitachi HD2700C Scanning Transmission Electron Microscope
}

\author{
H. Inada,* M. Konno,* Y. Suzuki,* K. Nakamura,* J. Wall,** and Y. Zhu***
}

* Nanotechnology Products Business Group, Hitachi High-Technologies Corp., Hitachinaka, Ibaraki 312-8504 Japan

** Biology department, Brookhaven National Laboratory, Upton NY 11973

*** Center for Functional Nanomaterials, Brookhaven National Laboratory, Upton NY 11973

With the ever shrinking size of nanostructured functional materials and devices the demand for better spatial resolution in atomic imaging and structure analysis has been soaring in recent years. To meet the demand, we developed a new model of scanning transmission electron microscope equipped with a CEOS Cs-corrector: Hitachi HD-2700C. One of the instruments was successfully installed and tested at Brookhaven's Center for Functional Nanomaterials. The instrument has a cold-field-emission electron source with high brightness and small energy spread [1]. The excellent electro-optical design and aberration correction make the instrument ideal for nanoprobe STEM imaging and energy-loss spectroscopy using transmitted electrons as well as for atomically resolved SEM using secondary and backscattered electrons to retrieve structural, chemical and bonding information of materials. The ability to image surface and bulk structure simultaneously at atomic resolution can revolutionize the field of microscopy and imaging [2].

The uniqueness of the HD-2700C is its capability of simultaneous signal acquisition for secondary electrons (SE) images and bright field (BF) and annular dark field (ADF) STEM images. The SE detector is installed above the pole piece, which is based on an Everhart-Thornley design, consisting of phosphor, crystal glass light guide, and photo multiplier (PMT) and associated electronics. A $10 \mathrm{kV}$ DC bias is applied to the phosphor to attract secondary electrons (Fig.1). Other detectors collecting transmitted and scattered electrons are located beneath the specimen and the collection angles for the detectors can vary by changing the projector lenses. To test and optimize the performance of the instrument, we have been working on high resolution imaging and spectroscopy of individual atoms and periodic crystals as well as on understanding the mechanism of the SEM imaging. Figure 2 are snap-shots, imaged at 200kV, of a specimen tilt series of $\mathrm{SrTiO}_{3}$ in both SEM and HAADF-STEM mode at the [001] zone axis (0 deg) and slightly tilted off zone axis (5 deg) to address the channeling effect on SE imaging. Figure 3 illustrates the atomic-number $(Z)$ dependence of SEM and HAADF-STEM image contrast for Uranium isolated atoms $\left(Z_{U}=92\right)$, Au nano particles $\left(Z_{\mathrm{Au}}=79\right), \mathrm{SrTiO}_{3}$ crystal $\left(\mathrm{Z}_{\mathrm{Sr}}=38, \mathrm{Z}_{\mathrm{TiO} 3}=30\right)$ and graphite Carbon $\left(\mathrm{Z}_{\mathrm{C}}=6\right)$ with Pt catalyst. Our preliminary results indicate that SEM imaging is capable of achieving atomic resolution for both heavy and light elements although the resolution for light elements may not be as good as the heavy elements. In comparison with STEM imaging, the SEM imaging seems more sensitive to light elements, yielding higher image intensity and contrast. The observations of atomic images of light elements were unexpected, and certainly challenged the traditional understanding of the SEM image formation mechanisms.

\section{References}

[1] Inada, H., Wu, L., Wall, J., Su, D., \& Zhu, Y., J. of Electron Microsc. 58, 111-122 (2009)

[2] Zhu, Y., Inada, H., Nakamura, K. \& Wall, J., Nature Mater. 8, 808-812 (2009) 


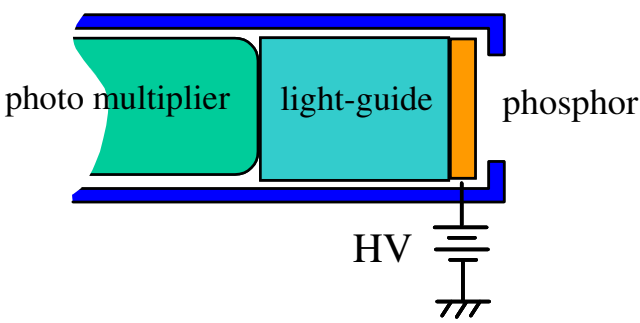

Figure 1. Schematic of the high efficiency Hitachi secondary electron detector, based on the Everhart-Thornley design.

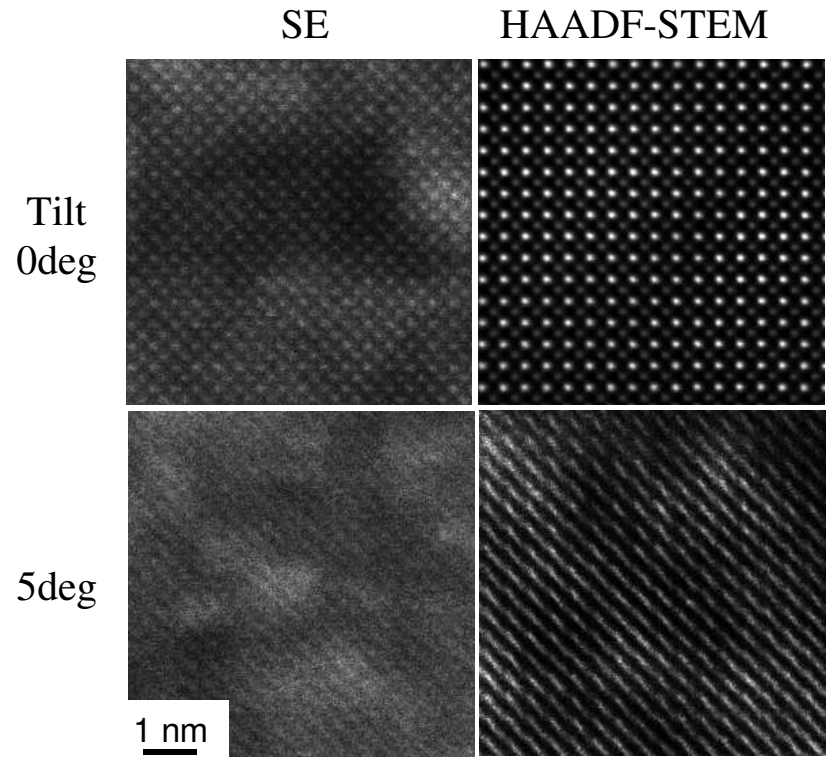

Figure 2. Comparison of two sets of SE and HAADF STEM images in a tilt series of $\mathrm{SrTiO}_{3}$ thin specimen to understand the channeling effect in SE imaging. Top row: in the (100) zone axis; bottom row: 5 degree away from the zone axis.

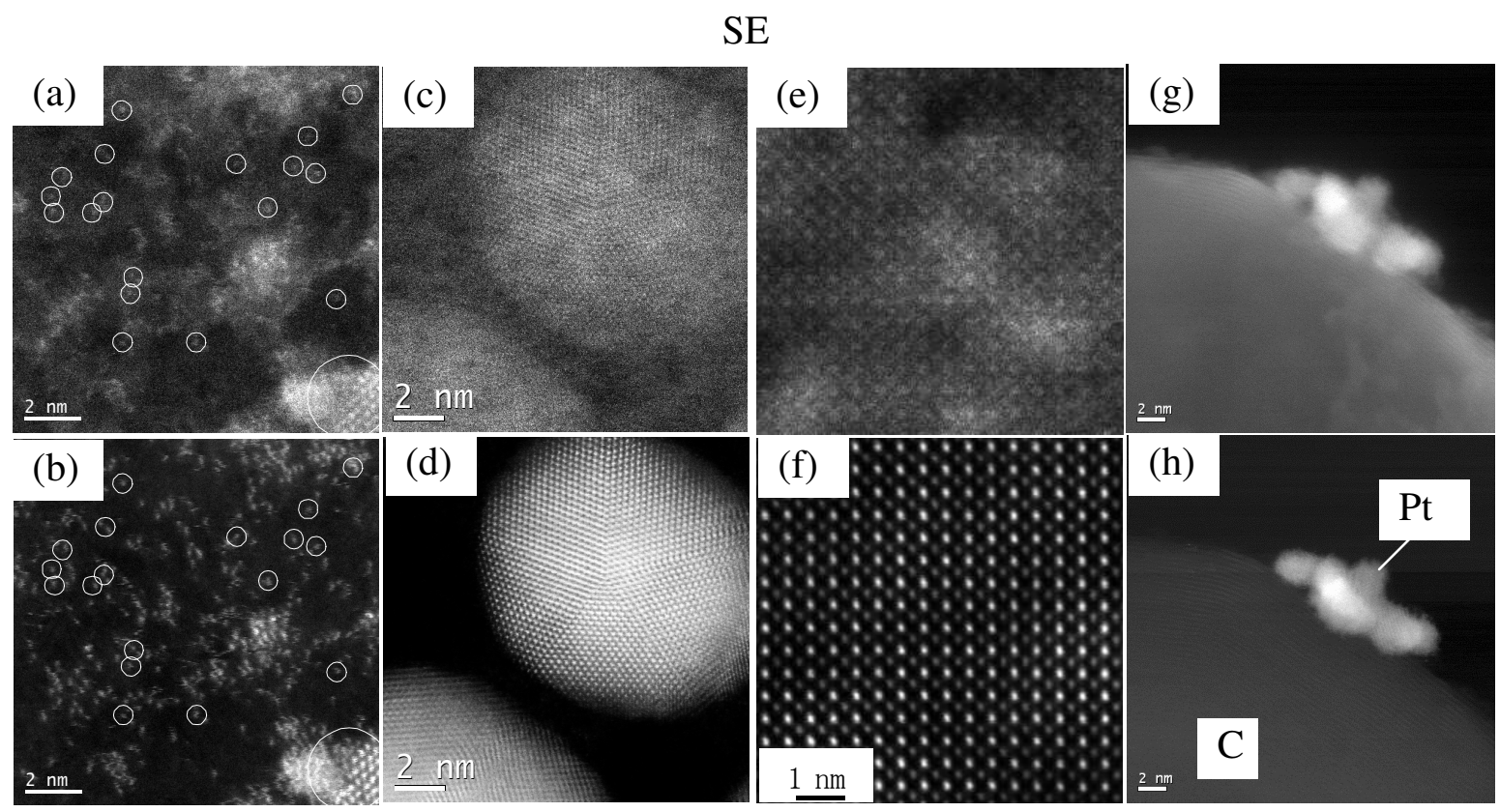

HAADF-STEM

Figure 3. Z-dependence of image contrast in SE imaging (top row) and HAADF-STEM imaging (bottom row). (a-b): Uranium atoms and nanocrystals (bottom corner) $\left(Z_{U}=92\right)$, (c-d): Au nano particles $\left(\mathrm{Z}_{\mathrm{Au}}=79\right)$, (e-f): $\mathrm{SrTiO}_{3}$ crystal $\left(\mathrm{Z}_{\mathrm{Sr}}=38, \mathrm{Z}_{\mathrm{TiO} 3}=30\right)$ and $(\mathrm{g}-\mathrm{h})$ : Pt catalyst sitting on graphite carbon $\left(\mathrm{Z}_{\mathrm{C}}=6\right)$. 